\title{
IMAGE RESTORATION USING HOS AND THE RADON TRANSFORM
}

\author{
Elisa Sayrol*, Chrysostomos L. Nikias*, and Toni Gasull ${ }^{* *}$ \\ *Signal and Image Processing Institute \\ University of Southern California \\ EEB 400 \\ Los Angeles CA 90089-2564 \\ tel: (213) 7404654, fax: (213) 7404651 \\ e-mail: sayrol@sipi.usc.edu \\ ** Department of Signal Theory and Communications. \\ Universitat Politecnica de Catalunya \\ Apdo. 30002 \\ 08080 Barcelona, Spain
}

\begin{abstract}
1
We propose the use of higher-order statistics (HOS) to study the problem of image restoration. We consider images degraded by linear or zero phase blurring point spread functions (PSF) and additive Gaussian noise. The complexity associated with the combination of twodimensional signal processing and higher-order statistics is reduced by means of the Radon Transform. The projection at each angle is an one-dimensional signal that can be processed by any existing I-D higherorder statistics-based method. We apply two methods that have proven to attain good one-dimensional signal reconstruction, especially in the presence of noise. After the ideal projections have been estimated, the Inverse Radon Transform gives the restored image. Simulation results are provided.
\end{abstract}

\section{INTRODUCTION}

Higher-Order statistics have been successfully applied before to the problem of 2-D signal reconstruction. In [1] the phase of the bispectrum is used to reconstruct images degraded by a jittery channel with additive tone interference. In [2] the bispectrum is used to estimate a randomly translating and rotating object from a sequence of noisy images. Another application is described in [3] where invariant Higher-Order Spectra features of projections are used for object classification.

The Bispectrum $B f\left(w_{1}, w_{2}\right)$ of a one-dimensional, deterministic, discrete-time signal $f(n)$, is defined as the Fourier Transform of its triple correlation function and is given by [4] [5]

$$
B\left(w_{1}, w_{2}\right)=F\left(w_{1}\right) F\left(w_{2}\right) F *\left(w_{1}+w_{2}\right),
$$

where $F(w)$ is the Fourier Transform of $f(n)$. The phase of the Bispectrum is therefore

\footnotetext{
$1_{\text {This work was supported by the Office of Naval Research under contract }}$ N00014-92-J-1034 and the Spanish Ministry of Education and Science.
}

$$
\angle B f\left(w_{1}, w_{2}\right)=\phi\left(w_{1}\right)+\phi\left(w_{2}\right)-\phi\left(w_{1}+w_{2}\right)(2)
$$

where $\phi(w)=\angle F(w)$. The Bispectra, and HOS in general, are insensitive to additive Gaussian noise and preserve the Fourier phase of the signal up to a linear phase factor. Our goal is to exploit such properties for the restoration of 2-D blurred and noisy images.

We have developed techniques that consider two HOS-based methods that have proven to attain good 1-D signal reconstruction. The first one is the Bispectrum Iterative Reconstruction Algorithm (BIRA) described in [6] that was utilized for signal reconstruction from the phase of the bispectrum. Although it was applied for restoration of images, the processing was made on a line by line basis. When the image is affected by a focus blur the distorting filter is 2-D and the reconstruction of lines does not lead to good results. This method cannot be extended to the two dimensional case since it is based on the cepstral coefficients that can not be defined in 2-D. Additionally, BIRA does not perform well when the Ztransform of the signal has zeros on the unit circle, unless an exponential window of the form $a^{n}$ moves the zeros outwards or inwards. An adequate parameter $a$ is usually difficult to find. Fortunately, as we will see, we can overcome both problems by reconstructing from projections. The second approach we study is the Weight Slice method (WS) [7] that recovers the signal of interest even in the case of zeros on the unit circle. However, although possible, its extension to 2-D will increase enormously its analytical complexity. We present an algorithm using the WS method over the projections of the image.

In Section 2 we describe the imaging system that we assume. We review the projection theorem that allows the restoration of images from their projections. In Section 3 we develop two different methods for the restoration either from the phase of the Bispectrum or from the phase of the Fourier Transform. In Section 4 some examples are given. Finally, Section 5 is devoted to conclusions and remarks. 


\section{PRELIMINARY DEFINITIONS}

To address the problem of recovering images from degraded versions of it, we assume a linear imaging system with space invariant point spread function and additive Gaussian noise. Thus, for the continuos model

$$
g(x, y)=\iint h\left(x-x^{\prime}, y-y^{\prime}\right) f\left(x^{\prime}, y^{\prime}\right) d x^{\prime} d y^{\prime}+n(x, y),
$$

where $g(x, y)$ is the observed image, $h(x, y)$ the point spread function, $f(x, y)$ the original image and $n(x, y)$ the additive Gaussian noise.

The 2-D signal recovery problem can be uniquely decomposed into many 1-D signal reconstruction problems. The Radon transform of a 2-D function, $f(x, y)$, denoted $f_{p} \theta(s)$, is defined as its line integral along a line inclined at an angle $\theta$ from the $y$-axis and at a distance $s$ from the origin. A fundamental relationship, the Projection Theorem, relates the 1-D Fourier transform of the projection $f_{p} \theta(s)$, with the central slice, at angle $\theta$, of the 2-D Fourier transform of the object $f(x, y)$

$g_{p \theta}(s)=h_{p \theta}(s) * f_{p \theta}(s) \leftarrow F_{1} s \rightarrow G(\theta, \xi)=H(\theta, \xi) F(\theta, \xi)$

where $g_{p} \theta(s), h_{p} \theta(s), f_{p} \theta(s)$ are the projections of $g(x, y)$, $h(x, y), f(x, y)$ at angle $\theta$, and $G(\theta, \xi), H(\theta, \xi), F(\theta, \xi)$ are the Fourier transforms in polar coordinates of $g(x, y)$, $h(x, y)$, and $f(x, y)$, respectively.

We consider that the Optical Transfer Function (OTF), the Fourier transform of the PSF, has linear or zero phase. As we see from (4), when the phase of the OTF is zero, the phase of the 1-D Fourier transform of each projection $G(\theta, \xi)$, corresponds to the phase of the Fourier transform of the projection of the original image, $F(\theta, \xi)$.

In most applications, blur degradations due to a number of sources are considered to have linear or zero phase. For example in [8] models for real out-of-focus images are investigated. It is shown that the phase is either zero or linear. Furthermore, a unique discrete representation results in a PSF support that covers the minimum number of pixels and where the OTF has zero phase.

It is well known that the discretization of the inverse formula for the Radon Transform is not easily defined. Finding accurate and fast algorithms is a current topic of research. On the other hand, if the PSF support is not an integer number, in terms of number of pixels, some distortion occurs. Despite these problems, restoration from projections is still feasible.

\section{RESTORATION FROM THE PHASE OF THE PROJECTIONS}

It was shown in [9] that a sequence of length $N$ can be uniquely specified if the phase function is known at (N-1) distinct frequencies in the interval $0<w<\pi$, except for a constant factor. Likewise in [6], it is shown that a sequence is uniquely specified if the phase of the bispectrum is known at $N(N-1) / 2$ distinct frequencies in the region $0<w_{1}+w_{2} \leq \pi, w_{2} \leq w_{1}, w_{1} \geq 0$, except for a constant factor and a linear shift. This is true under the assumptions that the Z-transform of the signal has not zeros in reciprocal pairs.

Therefore, if the projection of the blurring filter has length $d_{\theta}$ and the blurred projection has length $L_{\theta}$, there is a unique signal of length $L_{b \theta}=L_{\theta}-\left(d_{\theta}-1\right)$ with the same phase function (or phase bispectrum) than the blurred projection. This signal is nothing but the projection of the original image.

We next describe two HOS-based methods that can be used for the reconstruction of the projections. For more details on the algorithms see [6] and [7]

\subsection{Reconstruction with BIRA}

As we mentioned before, BIRA reconstructs a signal from the phase of its bispectrum. It employs the cepstral coefficients that for a signal $f_{p} \theta_{(n)}$ are defined as

$$
\begin{aligned}
& A_{p \theta}(m)=\sum_{i=1}^{L_{1}} a_{i p \theta} m \cdot \sum_{i=1}^{L_{2}} c_{i p \theta^{m}} \\
& B_{p \theta^{\prime}}(m)=\sum_{i=1}^{L_{3}} b_{i p \theta^{m}}
\end{aligned}
$$

where $a_{i p Q}, b i_{p} \theta_{\text {, }}$ are the zeros inside and outside the unit circle respectively, and $c_{i p} \theta$ are the poles inside the unit circle of the Z-transform of $f_{p} \theta(n)$. The last term is not included for finite sequences $\left(L_{2}=0\right)$. The cepstral coefficients are related to the phase of the bispectrum as

$$
C_{b f p} \theta^{(m, 0)}=-1 / m\left(A_{p \theta}(m)-B_{p \theta}(m)\right), m>0
$$

and to the power cepstrum as

$$
C_{p f p \theta}(m)=\left\{\begin{array}{cc}
-1 / m\left[A_{p \theta}(m)+B\left(p \theta^{m)}\right]\right. & m \infty 0 \\
\ln / A_{1 p \theta} / 2 & m=0 \\
-1 / m\left[A_{p \theta^{(-m)+B}}{ }_{p \theta}(-m)\right] & m<0
\end{array}\right.
$$

where $A_{1 p \theta}$ is a constant. Its relation to the signal is

$$
f_{p \theta^{(n)}}=F^{-1}\left(e^{F(C f p \theta(m))}\right), \quad n=0, . ., N-1
$$


where
$C_{f p \theta^{(m)}}=\left\{\begin{array}{cc}-1 / m A_{p \theta}(m) & m>0 \\ 0 & m=0 \\ 1 / m B_{p \theta}(-m) & m<0\end{array}\right.$

is the cepstrum of the signal.

The algorithm starts from the true sequence of the phase of the bispectrum for at least $N(N-1) / 2$ samples and an initial guess for the power cepstrum. It converges to a unique solution for the cepstral coefficients and consequently for the signal, except for a constant factor and a space shift. An additional procedure is added to control the space shift. The scale factor is calculated taking into account that the projection is a positive function and that all the projections have the same area, the volume of the image.

As we see in (5) and (6) in the case that there are zeros on the unit circle, the cepstral coefficients are infinite sequences. Its truncation leads to incorrect solutions. Two different alternatives are proposed when projections with zeros on the unit circle are detected.

A) When the number of such projections is low :

In this case we restore the image from the rest of the projections since the omission of few projections does not alter the restoration significantly.

B) When the number of such projections is high :

We try to identify the coefficients of the blurring filter using the following procedure:

B1) Reconstruct the projections that have neither zeros on the unit circle nor reciprocal pairs.

B2) From (4) we see that if we know $g p \theta(s)$ and $f p \theta(s)$ we can obtain $h p \theta(s)$ by deconvolution. The sequence is the projection of the blurring filter at an angle $\theta$ and its coefficients are weighted sums of the coefficients of the blurring filter. We build a system of equations of the following form

$$
X \boldsymbol{a}=\boldsymbol{y},
$$

where $X$ is the matrix of the weights of the coefficients at the specified angles, $\bar{a}$ is the coefficient vector and $y$ is the vector of the estimated blurring filter projected at the specified angles. As an example suppose we have only the projection at angle $\theta=0^{\circ}$ and a square $3 \times 3$ PSF with coefficients named from $a$ to $i$. Then, the system of equations will be

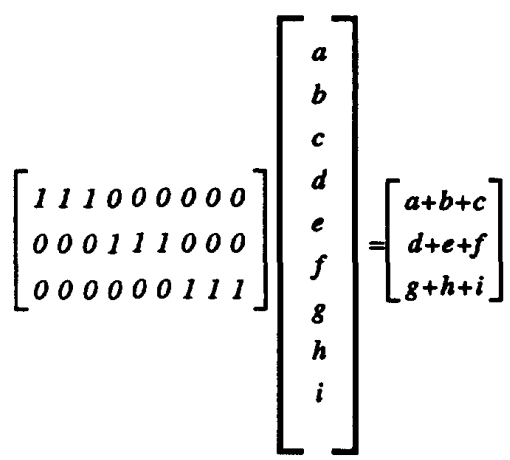

In general, we can solve the system of equations to find the coefficient vector $\boldsymbol{a}$ if the rank of the matrix is equal or greater than $N_{c}$. Using a LS approximation the solution will be

$$
a=\left(X^{H} X\right)^{-1} X^{H} y
$$

B3) Once we know the blurring filter coefficients we can either deconvolve the 2-D noise-free image or the 1d noise-free projections.

\subsection{Reconstruction with the WS method}

We can uniquely reconstruct the projections from the phase only of the Fourier transform even in the case zeros lie on the unit circle [9]. However, in the presence of noise the phase is degraded. Therefore, we decompose the reconstruction of a blurred noisy projection in two steps. In the first step, a method based on HOS reduces Gaussian noise preserving the phase function. We choose the WS method because it works when there are roots on the unit circle and it has better performance than other methods. In the second step we deblur the signal from its phase.

Step 1) It is known that for a causal and exponentially stable system with input assumed to be independent, identically distributed and non Gaussian, with skewness $\beta$, the output bispectrum $B_{\mathcal{X}}\left(w_{1}, w_{2}\right)$ exists and is given by [3], [4]

$$
B_{x}\left(w_{1}, w_{2}\right)=\beta H\left(w_{1}\right) H\left(w_{2}\right) H^{*}\left(w_{1}+w_{2}\right)
$$

As we see, finding the coefficients of the filter $H(w)$ is equivalent to estimate the sequence $F(w)$ in (1).

The Weight Slice (WS) algorithm has been previously used to obtain the parameters of a non-minimum FIR system. The parameters can be expressed as a linear combination of cumulant slices. It was shown that if there exists a set of weights that gives a causal slice, then the FIR system can be identified. The system of equations is expressed as

$$
S w=b_{o}
$$

where $S$ is the matrix of cumulants 


$$
S=\left[\begin{array}{cccc}
c_{2 x^{(-q)}} & c_{3 x^{(-q, j)}} & c_{4 x^{(-q, j, k)}} & - \\
\cdot & \cdot & \cdot & \cdot \\
c_{2 x^{(0)}} & \cdot & \cdot & \cdot \\
\cdot & \cdot & \cdot & \cdot \\
c_{2 x^{(q)}} & \cdot & \cdot & \cdot
\end{array}\right]
$$

$C_{n x}$ is the estimated $n^{\text {th }}$ order cumulant of the output signal, $w$ is the weight vector

$$
w=(w 2, w 3(j), w 4(j, k), \ldots) t
$$

and $b_{o}$ is the coefficient vector

$$
b_{0}=(0 \ldots 01 \ldots b(q-1) b(q)) \text {. }
$$

The unknowns are the vector $w$, and the last $q$ elements of $b_{o}$.

The matrix equation is solved in two steps :

1) Computation of the minimum norm weights that give a causal W-slice

$$
\begin{gathered}
S_{u} w_{m}=(0, \ldots, 0,1) t \\
w_{m}=S_{u}^{\# 1}
\end{gathered}
$$

where $S u$ is the matrix formed from the upper $q+1$ rows of $S$, and $S_{u}^{\#}$ denotes the pseudo inverse of $S_{u}$.

2) Computation of the coefficients as

$$
b_{o}=S w_{m}=S S_{u}^{\# 1}
$$

Step 2) The vector $b_{o}$ is the sequence of the noisereduced blurred projection. Once $b_{o}$ has been calculated, we deblur the signal using a similar algorithm than the one described in [9]. It is equivalent to BIRA, it starts with the true sequence of the phase of the Fourier Transform and an initial guess for its magnitude.

\section{EXAMPLES}

In the next examples, the Radon Transform is calculated from an interpolation of the Cartesian sampling grid of the 2-D FFT of the image to a polar grid. The Inverse Radon Transform is implemented in a reverse way.

Fig. 1a shows a $24 \times 24$ image. The reconstructed image after applying Radon Transform followed by the Inverse Radon transform is shown on Fig. 1d. The dimensions of the 2-D FFT and 2-D IFFT are $64 \times 64$ to avoid distortion in the high frequencies. Fig. $1 \mathrm{~b}$ presents a blurred version of this image when a $3 \times 3$ Gaussian filter with parameter $\alpha=0.4$ is used. Fig. le shows the restored image from the Fourier phase of the projections. In Fig. $1 \mathrm{c}$ the image is blurred with the same filter and Gaussian noise for SNR=25 dbs is added. The restored image is shown in Fig. If where the Ws method is applied using one slice only, the third order cumulant is estimated from 50 realizations. Another example is shown in Fig. 2a and Fig. 2d, where the original image is $64 \times 64$ and the 2-D FFTs are $256 \times 128$. Fig. $2 b$ is a blurred version of this image with a $5 \times 5$ Gaussian filter and $\alpha=0.4$. Fig. $2 \mathrm{c}$ is the restored image from the Fourier phase. From this image we see that high frequencies have been recovered. However, we observe some distortion. This is due to relative shifting among projections. Fig. 3 shows a typical projection, its blurred version, the reconstructed signal and the difference between the original and the restored signal.

\section{CONCLUSIONS AND REMARKS}

In this paper, we have shown that it is possible to restore a blurred and noisy image from HOS of its projections. Two different approaches were given to reconstruct the 1D signals from either the phase of the Fourier Transform or the phase of the bispectrum. Although the complexity using HOS over 2-D signals is reduced, the computational load is still high. Some other applications of HOS over the projections are under investigation.

\section{REFERENCES}

[1] S.A. Dianat, M. R. Raghuveer, "Fast Algorithms for phase and magnitude reconstruction from bispectra", Optical Engineering,May 1990.

[2] B.M.Sadler, "Shift and Rotation Invariant Object Reconstruction using Bispectrum", Workshop on HOS analysis, Vail, Colorado, 1989,pag 106

[3] V. Chandran, S. Elgar, "Position, rotation, and scale invariant recognition of images using Higher-order spectra", Proc. ICASSP '92, S.F.,pp v-213-216.

[4] C. L. Nikias, M. R. Raghuveer,"Bispectrum estimation : A Digital Signal Processing Framework", Proc. of IEEE, V-75, No. 7, July 87.

[5] J.M. Mendel, "Tutorial on Higher-Order Statistics (Spectra) in signal processing and system theory: Theoretical results and some applications", Proc. of IEEE, Vol 79, No. 3, Nov. 1991.

[6] A. Petropulu, C. L. Nikias, "Signal Reconstruction from the phase of the Bispectrum", IEEE Transactions on Signal Processing, Vol. 40, No. 3, pag 601 .

[7] J.A. Fonollosa, J. Vidal, "System Identification using a linear combination of cumulant slices", submitted to IEEE Transactions on Signal Processing.

[8] M.I. Sezan, G. Pavlovic, M., Tekalp, "On modeling the focus blur on Image restoration", Proc. ICASSP '92, Albuquerque, NM, pp 2485-2488.

[9] M. H. Hayes, J. S. Lim and A. V. Oppenheim. "Signal Reconstruction from the phase or magnitude", IEEE on ASSSP, Vol 28, No. 6, Dec 1980. 


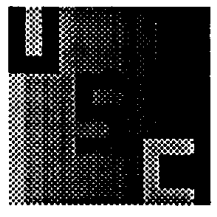

Fig. 1a

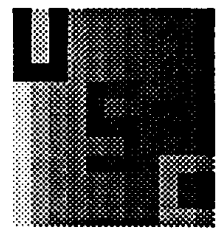

Fig. 1d

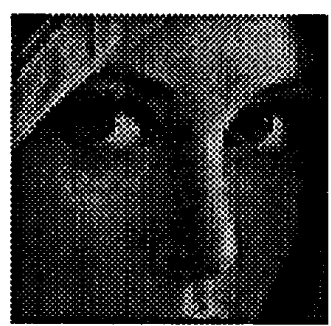

Fig. 2a

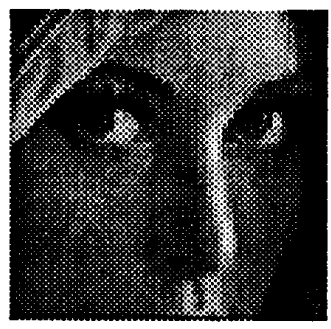

Fig. 2d

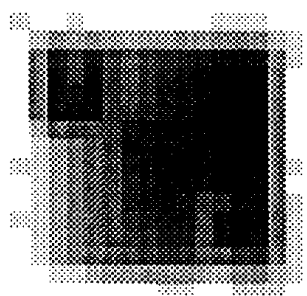

Fig. 1b

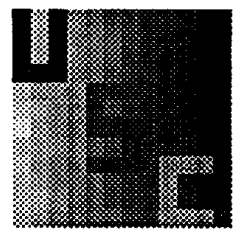

Fig. 1e

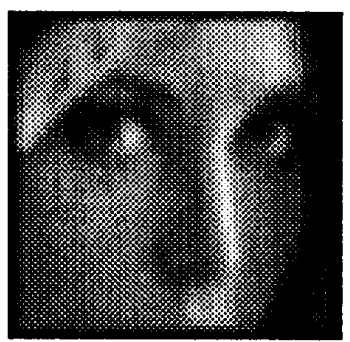

Fig. 2b
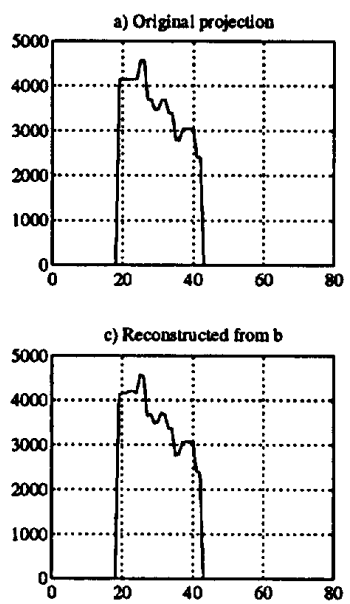

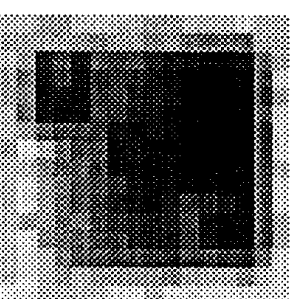

Fig. 1c

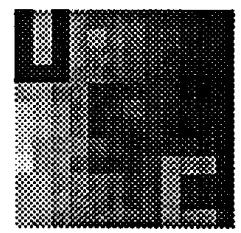

Fig. 1f

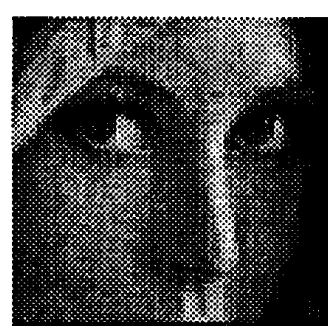

Fig. 2c
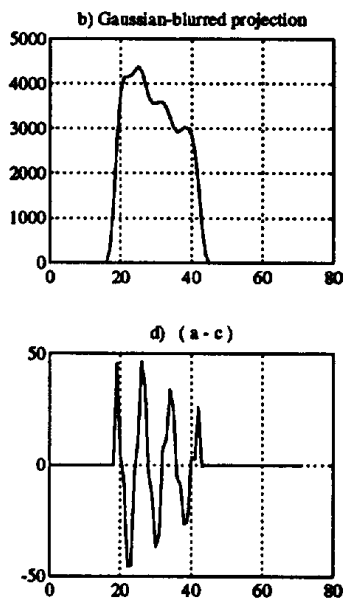